\title{
Parâmetros reprodutivos de cabras Toggenburg inseminadas com sêmen resfriado, após diluição em meio à base de gema de ovo
}

[Reproductive parameters of Toggenburg goats inseminated with cooled semen diluted in egg yolk extender]

\author{
A.P. Siqueira ${ }^{1}$, J.F. Fonseca ${ }^{2}$, J.M. Silva Filho ${ }^{3 *}$, J.H. Bruschi ${ }^{4}$, J.H.M. Viana ${ }^{4}$, M.S. Palhares ${ }^{3}$, \\ M.C.M. Bruschi ${ }^{5}$, M.P. Peixoto ${ }^{5}$ \\ ${ }^{1}$ Aluno de pós-graduação - EV-UFMG - Belo Horizonte, MG \\ ${ }^{2}$ Embrapa Caprinos - Sobral, CE \\ ${ }^{3}$ Escola de Veterinária - UFMG \\ Caixa Postal 567 \\ 30123-970 - Belo Horizonte, MG \\ ${ }^{4}$ Embrapa Gado de Leite - Coronel Pacheco, MG \\ ${ }^{5}$ Médica veterinária autônoma
}

\begin{abstract}
RESUMO
Avaliaram-se a taxa de concepção, a resposta à prostaglandina, a duração do estro, a categoria reprodutiva e o tipo de muco de cabras inseminadas com sêmen diluído em meio à base de gema de ovo e resfriado a $5^{\circ} \mathrm{C}$, por 12 ou 24 horas. Foram utilizados dois reprodutores e 62 fêmeas da raça Toggenburg, que receberam duas doses de 22,5 $\mu$ g de PGF2 $\alpha$, em intervalos de 10 dias, para a sincronização do estro. A partir da primeira aplicação de PGF2 $\alpha$, o estro foi monitorado três vezes ao dia. Realizou-se uma única inseminação, 12 horas após o início do estro. As porcentagens de fêmeas em estro, após a primeira e segunda aplicações de PGF $2 \alpha$, foram de $85,5 \%$ e $88,7 \%$, respectivamente. O intervalo de aplicação da primeira e segunda doses de $\mathrm{PGF}_{2} \alpha$ ao início do estro foi

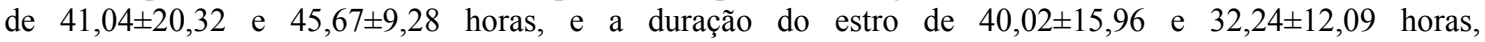
respectivamente. A taxa de concepção total foi de $49,1 \%$. O período de armazenamento do sêmen e a categoria reprodutiva não influenciaram $(\mathrm{P}>0,05)$ a taxa de concepção. O tipo de muco observado no momento da inseminação influenciou $(\mathrm{P}<0,05)$ a fertilidade das fêmeas, sendo o de aspecto estriado associado aos maiores índices de concepção.
\end{abstract}

Palavras-chave: caprino, sêmen resfriado, inseminação artificial, taxa de concepção

\begin{abstract}
The conception rate, the prostaglandin response, the estrus duration, the reproductive class, and the mucous of goats inseminated with semen diluted in egg yolk extender and cooled at $5^{\circ} \mathrm{C}$, for 12 or 24 hours were evaluated. Sixty-two female goats and two sexually mature Toggenburg bucks were used. The females received two doses of $22.5 \mu \mathrm{g}$ of prostaglandine F2 $\alpha$, at 10-day intervals. After the first injection, the estrus was monitored three times a day (6:00, 12:00, and 18:00h), with a buck teaser. Only one insemination was used. The percentages of animals that showed estrus after the first and the second injection of PGF $2 \alpha$ were $85.5 \%$ and $88.7 \%$, respectively. The average intervals from first and second PGF2 $\alpha$ injection to estrus were $41.04 \pm 20.32$ and $45.67 \pm 9.28 \mathrm{~h}$, and the estrus durations for both injections were $40.02 \pm 15.96$ and $32.24 \pm 12.09 \mathrm{~h}$, in this order. The interval from the PGF2 $\alpha$ injection to the beginning of the estrus was longer $(P<0.05)$, whereas the estrus duration was shorter $(P<0.05)$ in the estrus induced after the second $P G F 2 \alpha$ injection. The total conception rate was $49.1 \%$. Neither the semen storage length (12 or 24 hours) nor the reproductive class did not affect $(P>0.05)$ the conception rate. The mucous observed at the insemination time influenced $(P<0.05)$ the fertility of inseminated goats, with the striated aspect associated to higher fertility.
\end{abstract}

Keywords: goat, cooled semen, artificial insemination, conception rate

Recebido em 4 de janeiro de 2008

Aceito em 9 de fevereiro de 2009

* Autor para correspondência (corresponding author)

E-mail: monteiro@vet.ufmg.br

Apoio: FAPEMIG (CAG n ${ }^{\circ}$ 1243/05) 


\section{INTRODUÇÃO}

A inseminação artificial representa uma biotecnologia de reprodução assistida que possibilita a utilização de indivíduos geneticamente superiores, associada a menores custos por serviço. Além disso, elimina a necessidade de transporte dos animais, diminui os custos e os inconvenientes da manutenção de um grande número de machos na propriedade, e permite registros precisos sobre a reprodução do rebanho, o que possibilita a realização de programas de acasalamento e o controle de determinadas enfermidades. Associada ao teste de progênie, permite a identificação mais acurada dos reprodutores, tornando-se importante ferramenta para o melhoramento genético da espécie caprina (Siqueira, 2006).

A sincronização do estro tem sido amplamente usada em caprinos como ferramenta auxiliar nos programas de inseminação (Alves et al., 2005). A indução e a sincronização do estro e da ovulação são importantes práticas de controle reprodutivo, principalmente quando aplicadas em animais estacionais como os caprinos. Elas permitem a manipulação reprodutiva, concentrando as atividades do rebanho, como a monta natural ou inseminação artificial e a estação de nascimento, facilitando o manejo reprodutivo. Além disso, permitem um planejamento da produção e da oferta de carne e leite (Fonseca, 2002; Maffilli et al., 2006; Amorim et al., 2008). A sincronização pode ser obtida pela redução da fase lútea, com a aplicação de prostaglandinas, em dose única ou dupla, intervaladas de 11 dias, ou prolongando a duração da fase lútea, utilizando-se uma combinação de progestágenos, gonadotrofinas e prostaglandinas, em caso de fêmeas em anestro sazonal (Fonseca, 2002).

A observação do muco, e da sua alteração durante o período de cio, pode ser usada para estimar o melhor momento do estro para se realizar a inseminação, uma vez que há correlação entre o tipo de muco observado no momento da inseminação e a taxa de fertilidade. Dessa forma, o melhor momento para se realizar a inseminação é quando o muco apresenta-se estriado e abundante, 12 a 18 horas após o início do estro. Taxas de parto de 76,2 e $86,4 \%$ foram observadas em cabras inseminadas quando o muco cervical apresentava-se com aspecto cristalino e estriado, respectivamente (França,
1981). Entretanto, quando a inseminação é realizada na presença de muco com aspecto cristalino, recomenda-se uma segunda inseminação, 12 a 14 horas após a primeira (Bonfert, 1964, citado por Simplício, 1987).

O local de deposição do sêmen durante a inseminação também tem importância relevante na taxa de concepção e está diretamente relacionado à categoria reprodutiva (Barbosa, 1999). Azevedo et al. (1995) enfatizaram a dificuldade de se realizar a deposição do sêmen em locais mais profundos da genitália de cabras nulíparas com o método transcervical. Corteel (1997) relatou que a taxa de concepção, na inseminação artificial, é afetada de maneira proporcional ao grau de dificuldade em transpor a cérvice. Azevedo et al. (1998) mencionaram que, além da maior dificuldade em transpor a cérvice em cabras nulíparas, há também maior nível de estresse nos animais durante as inseminações, o que pode influenciar negativamente a concepção.

O objetivo deste estudo foi avaliar os parâmetros reprodutivos de cabras inseminadas com sêmen resfriado a $5^{\circ} \mathrm{C}$, submetidas à sincronização do estro.

\section{MATERIAL E MÉTODOS}

Detalhes sobre o local e o período experimental, assim como o manejo e a alimentação dos animais, foram descritos por Siqueira et al. (2009).

Foram utilizadas 62 fêmeas da raça Toggenburg, de diferentes categorias reprodutivas, cabritas e cabras secas ou lactantes e, como doadores de sêmen, dois machos sexualmente maduros, pertencentes à mesma raça.

As fêmeas foram distribuídas uniformemente, de acordo com a categoria reprodutiva, em dois tratamentos $(\mathrm{T})$ : no T1, o sêmen foi resfriado a $5^{\circ} \mathrm{C}$, e a inseminação ocorreu após 12 horas de estocagem; no $\mathrm{T} 2$, o sêmen foi resfriado a $5^{\circ} \mathrm{C}$ e a inseminação ocorreu após 24 horas de estocagem. Para a indução do estro, receberam duas aplicações de $22,5 \mu \mathrm{g}$ de prostaglandina sintética, d-cloprostenol ${ }^{1}$, na submucosa vulvar, em intervalos de 10 dias. Após a primeira

${ }^{1}$ Prolise ${ }^{\circledR}$ (fco $20 \mathrm{~mL}$ ) - Tecnopec - São Paulo, Brasil. 
aplicação de PGF2 $\alpha$ e até o dia do diagnóstico da gestação pela ultra-sonografia, os animais foram monitorados três vezes ao dia, às 6,12 e $18 \mathrm{~h}$, quanto ao início do estro, com auxílio de um rufião cirurgicamente preparado. Considerou-se como início do cio a primeira aceitação de monta pelo rufião.

A coleta de sêmen foi realizada pelo método da vagina artificial, segundo Fonseca $(2005)^{2}-$ comunicação pessoal - e descrito por Siqueira et al. (2009). Após a coleta, o sêmen foi diluído em Tris-frutose-gema de ovo a 2,5\% (Evans e Maxwell, 1987), envasado em palhetas de $0,25 \mathrm{~mL}$, com $150 \times 10^{6}$ espermatozoides móveis e resfriado a $5^{\circ} \mathrm{C}$. O sêmen foi conservado em container apropriado (Palhares, 1997), até o momento da inseminação, após 12 ou 24 horas de armazenamento a $5^{\circ} \mathrm{C}$.

Foi realizada uma única inseminação, pelo método de fixação da cérvice, 12 horas após a aceitação da monta. Foram inseminadas somente as cabras que responderam, até o quarto dia, à aplicação da segunda dose de PGF2 $\alpha$. Dessa forma, as cabras que entraram em cio após esse período não foram inseminadas.

O diagnóstico de gestação foi feito aos 21, 30 e 60 dias, por ultra-sonografia transretal ${ }^{3}$. Também se considerou o monitoramento diário do retorno ao cio.

Estudaram-se: taxas de concepção - taxa de concepção total, taxa de prenhez por tipo de muco, taxa de prenhez por categoria reprodutiva, taxa de prenhez por bode e taxa de prenhez por tratamento -, assim como o horário de início do estro, o tipo de muco, a categoria reprodutiva e o local de deposição do sêmen por tratamento, usando o pacote estatístico SÃS/1990 - e o teste do qui-quadrado para avaliação das características qualitativas.

Os dados quantitativos referentes ao intervalo entre as aplicações de PGF2 $\alpha$ e o início do estro, duração do estro, tempo da coleta à inseminação, intervalo do início do estro à inseminação, duração da inseminação, intervalo da IA ao término do estro e retorno ao estro após a IA foram submetidos à análise de variância e ao teste $\mathrm{t}$, para comparação de duas médias, e ao teste SNK, para comparação de mais de duas médias (Sampaio, 2002).

Os resultados foram apresentados considerandose parâmetros de controle e parâmetros de resultados. Os parâmetros de controle são aqueles que informam sobre a homogeneidade dos tratamentos, não sendo resultados destes, enquanto os parâmetros de resultados representam respostas aos tratamentos utilizados.

Consideraram-se como parâmetros de controle o número de cabras, a idade, o escore corporal e o peso, e, como parâmetros de resultados, o intervalo da segunda aplicação de $\mathrm{PGF}_{2} \alpha$ ao início do estro, a duração do estro após segunda aplicação de $\mathrm{PGF}_{2} \alpha$, o tempo da coleta do sêmen à IA, o intervalo do início do estro à IA, a duração da IA, o intervalo da IA ao término do estro, a taxa de concepção e o retorno ao estro após a IA.

\section{RESULTADOS E DISCUSSÃO}

Não se observou influência $(\mathrm{P}>0,05)$ de tratamento sobre os parâmetros reprodutivos das cabras inseminadas com sêmen resfriado a $5^{\circ} \mathrm{C}$ por 12 ou 24 horas (Tab. 1).

Não houve diferenças $(\mathrm{P}>0,05)$ quanto às taxas de concepção obtidas com uma única inseminação, 12 horas após a identificação do estro, entre os períodos de armazenamento do sêmen com $12(55,6 \%)$ ou 24 horas $(42,9 \%)$, a $5^{\circ} \mathrm{C}$.

Resultados consistentes foram relatados por Roca et al. (1997) ao utilizarem sêmen não lavado, preservado em meio diluidor contendo $2 \%$ de gema de ovo e resfriado a $5^{\circ} \mathrm{C}$, quando obtiveram taxa de concepção de $73,5 \%$, porém após duas inseminações. Além disso, a concentração/dose inseminante utilizada por esses pesquisadores foi de $240 \times 10^{6} \mathrm{sptz} / \mathrm{mL}$, enquanto a utilizada no presente experimento foi de $150 \times 10^{6} \mathrm{sptz} / 0,25 \mathrm{~mL}$. Há que se enfatizar que a realização de mais de uma inseminação possibilita fazer esse procedimento em um momento mais próximo da ovulação.

${ }^{2}$ Fonseca, J.F., 2005. Embrapa Caprinos - Sobral, CE.

${ }^{3}$ Aloka, modelo SSD - 500 - Tokyo, Japão. 
Tabela 1. Parâmetros reprodutivos de cabras inseminadas com sêmen diluído em extensor à base de gema de ovo e resfriado a $5^{\circ} \mathrm{C}$, por 12 ou 24 horas

\begin{tabular}{lcc}
\hline & $\begin{array}{c}\text { 12h de armazenamento } \\
(\mathrm{n})\end{array}$ & $\begin{array}{c}\text { 24h de armazenamento } \\
(\mathrm{n})\end{array}$ \\
\hline Parâmetro de controle & 27 & 28 \\
\hline Número de cabras & $40,27 \pm 27,81(22)$ & $40,14 \pm 24,51(22)$ \\
Idade (meses) & $3,46 \pm 0,74(26)$ & $3,49 \pm 0,58(25)$ \\
Escore corporal (1-5) & $44,41 \pm 8,85(27)$ & $47,22 \pm 8,41(28)$ \\
Peso (kg) & & \\
\hline Parâmetro de resultado & $45,33 \pm 9,93(27)$ & $47,14 \pm 10,51(28)$ \\
\hline Intervalo 2 ${ }^{\mathrm{a}} \mathrm{PGF}_{2} \alpha-$ estro (h) & $33,67 \pm 12,18(27)$ & $31,07 \pm 12,36(28)$ \\
Duração do estro após 2 PGF $_{2} \alpha(\mathrm{h})$ & $12,09 \pm 1,95(27)$ & $23,99 \pm 2,26(28)$ \\
Tempo da coleta à IA (h) & $19,77 \pm 6,41(27)$ & $17,83 \pm 5,07(28)$ \\
Intervalo início do estro $-\mathrm{IA}(\mathrm{h})$ & $72,19 \pm 55,10(27)$ & $62,52 \pm 55,61(27)$ \\
Duração da IA (seg) & $13,69 \pm 12,13(27)$ & $13,02 \pm 14,07(28)$ \\
Intervalo IA ao término do estro (h) & $55,56(15 / 27)$ & $42,86(12 / 28)$ \\
Taxa de concepção (\%) & $11,28 \pm 7,88(11)$ & $12,89 \pm 8,33(16)$ \\
Retorno ao estro após a IA (dias) & & \\
\hline
\end{tabular}

Quanto ao momento da inseminação (Tab. 1), após avaliação da duração do estro, do intervalo do início do estro à IA e do intervalo da IA ao término do estro, observa-se que a IA foi realizada, na maioria dos casos no momento adequado, próximo ao terço final do estro. Como neste trabalho as cabras foram inseminadas, em média, 19,02 $\pm 5,66$ horas do início do estro, provavelmente as que apresentaram estro de longa duração foram inseminadas muito precocemente, ao contrário das com estro muito curto, cujas inseminações foram realizadas tardiamente. Essas duas situações podem ter influenciado os resultados de fertilidade obtidos neste experimento, principalmente se se considerar a utilização de apenas uma inseminação por ciclo. Em muitos experimentos, é difícil determinar a verdadeira influência do momento da inseminação sobre a taxa de gestação, uma vez que vários fatores podem interferir no resultado. Dentre eles, vale salientar o método de preservação do sêmen, a concentração espermática e o volume da dose inseminante, a duração do estro e o local de deposição do sêmen no sistema genital feminino.

Das 62 cabras submetidas à sincronização com $\mathrm{PGF}_{2} \alpha$, seis $(9,7 \%)$ não responderam à segunda aplicação e, portanto, não foram inseminadas. Uma cabra respondeu à segunda dose de prostaglandina e foi inseminada, mas persistiu em cio por 225 horas, sendo, por isso, retirada do experimento.

Houve influência $(\mathrm{P}<0,05)$ da aplicação de $\mathrm{PGF}_{2} \alpha$ sobre o intervalo $\mathrm{PGF}_{2} \alpha$-estro e sobre a duração do estro (Tab. 2). Assim, os maiores valores $(\mathrm{P}<0,05)$ referentes ao intervalo $\mathrm{PGF}_{2} \alpha$ estro foram observados após a segunda aplicação. A duração do estro foi menor $(\mathrm{P}<0,05)$, e o número de animais que entrou em estro foi maior após a aplicação da segunda dose de $\mathrm{PGF}_{2} \alpha(88,7 \%$ vs $85,5 \%)$. De acordo com Menchaca e Rubianes (2004), a variabilidade na resposta aos tratamentos com $\mathrm{PGF}_{2} \alpha$ pode estar relacionada à condição folicular de cada animal, no momento da administração da $\mathrm{PGF}_{2} \alpha$. Se um folículo grande, em crescimento, está presente no momento do tratamento, ele continuará seu desenvolvimento, com o estro e ovulação ocorrendo em curto período de tempo, após a administração da $\mathrm{PGF}_{2} \alpha$. Contudo, se a luteólise for induzida quando o maior folículo da onda estiver em regressão, um novo folículo precisa emergir e crescer, e, neste caso, o estro e a ovulação podem ocorrer mais tarde. 
Tabela 2. Intervalo (média $\pm \mathrm{DP}$ ) do tratamento com $\mathrm{PGF}_{2} \alpha$ e início do estro e duração do estro, em horas, após a aplicação de duas doses de prostaglandina, intervaladas de 10 dias

\begin{tabular}{lcc}
\multicolumn{1}{c}{ Variável } & $1^{\underline{a}}$ aplicação de PGF $_{2} \alpha(\mathrm{n})$ & $2^{\underline{a}}$ aplicação de PGF2 $\alpha(\mathrm{n})$ \\
\hline Intervalo $\mathrm{PGF}_{2} \alpha$ - estro (h) & $41,04 \pm 20,32 \mathrm{a}(53)$ & $45,67 \pm 9,28 \mathrm{~b}(55)$ \\
\% manifestação do estro & $85,48(53 / 62)$ & $88,71(55 / 62)$ \\
Duração do estro (horas) & $40,02 \pm 15,96 \mathrm{a}(53)$ & $32,24 \pm 12,09 \mathrm{~b}(55)$ \\
\hline
\end{tabular}

Médias na mesma linha seguidas de letras distintas diferem entre si $(\mathrm{P}<0,05)$ pelo teste t. DP: desvio-padrão.

Ott et al. (1980), ao utilizarem duas doses de $\mathrm{PGF}_{2} \alpha$, intervaladas de 11 dias, observaram $70,6 \%$ de manifestação de estro em um período de $54 \pm 3$ horas após a aplicação da primeira dose de prostaglandina. Além disso, 94\% dos animais manifestaram estro $52 \pm 3$ horas após a segunda aplicação.

Resultados semelhantes foram obtidos por Fonseca (2002), que observou manifestações de estro em $73,7 \%$ e $89,5 \%$ das cabras após a aplicação da primeira e da segunda dose de prostaglandina, respectivamente. Esse autor não observou diferenças nos intervalos entre as aplicações de prostaglandina e o início do estro $(44,5 \pm 15,9$ horas na primeira aplicação e $49,9 \pm 11,9$ horas na segunda aplicação). No mesmo estudo, verificou, também, redução na duração do estro $(\mathrm{P}<0,05)$, após a segunda aplicação (16,2 $\pm 10,8$ horas), em relação à observada após a primeira dose de $\mathrm{PGF}_{2} \alpha$ $(31,7 \pm 11,1$ horas $)$.

Romano (1994), ao estudar a influência de vários estímulos sobre a duração do estro, observou que a monta e a estimulação mecânica da vagina reduziram significativamente a duração do estro $(22,8 \pm 5,0$ horas e $27,6 \pm 6,8$ horas,

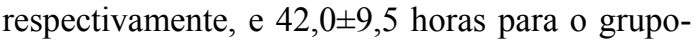
controle). Dessa forma, o resultado observado neste estudo encontra-se dentro do esperado, uma vez que diferentes estímulos realizados durante a inseminação, a saber: a distensão vaginal causada pelo espéculo, o estímulo cervical pelo cateter e o plasma seminal presente na dose inseminante podem ter respondido, isolada ou aditivamente, pela redução na duração do estro após a segunda aplicação de $\mathrm{PGF}_{2} \alpha$, exatamente os estros acompanhados por inseminações.

Não foi observado efeito $(\mathrm{P}>0,05)$ da categoria reprodutiva sobre a taxa de concepção das cabras inseminadas com sêmen diluído e resfriado a $5^{\circ} \mathrm{C}$ (Tab. 3).
Tabela 3. Taxa de concepção das cabras inseminadas com sêmen diluído e resfriado a $\underline{5^{\circ} \mathrm{C} \text {, segundo a categoria reprodutiva }}$

\begin{tabular}{lc}
\hline Categoria reprodutiva & $\begin{array}{c}\text { Taxa de } \\
\text { concepção (\%) }\end{array}$ \\
\hline Cabrita & $54,55(06 / 11)$ \\
Cabra seca & $39,29(11 / 28)$ \\
Cabra lactante & $64,29(09 / 14)$ \\
Total & $49,06(26 / 53)$ \\
\hline
\end{tabular}

A taxa de concepção foi influenciada $(\mathrm{P}<0,05)$ pelo tipo de muco observado no momento da inseminação (Tab. 4). Além disso, 60\% das inseminações foram realizadas quando o muco apresentava-se estriado, evidenciando o momento ideal no qual se realizaram as inseminações. A observação do muco e da modificação de sua aparência durante o período de estro pode ser usada para estimar o melhor momento do estro para se realizar a inseminação, uma vez que houve influência do tipo de muco, observado no momento da inseminação, sobre a taxa de concepção das cabras.

Tabela 4. Taxa de concepção de cabras inseminadas com sêmen diluído e resfriado a $5^{\circ} \mathrm{C}$, segundo o tipo de muco

\begin{tabular}{lc}
\hline \multicolumn{1}{c}{ Tipo de muco } & $\begin{array}{c}\text { Taxa de } \\
\text { concepção }\end{array}$ \\
\hline Cristalino & $15,38(2 / 13) \mathrm{a}$ \\
Cristalino-estriado* & $0,00(0 / 1)$ \\
Estriado & $57,58(19 / 33) \mathrm{b}$ \\
Estriado-caseoso & $80,00(4 / 5) \mathrm{b}$ \\
Caseoso & $66,67(2 / 3) \mathrm{ab}$ \\
Total & $49,09(27 / 55)$ \\
\hline
\end{tabular}

Médias na mesma coluna seguidas por letras distintas diferem entre si $(\mathrm{P}<0,05)$ pelo teste SNK.

* Retirada da análise estatística por ter apenas uma fêmea no grupo

O melhor momento para se realizar a inseminação é quando o muco apresenta-se estriado e abundante, o que corresponde ao terço médio para o final do estro (12-18 horas após o seu início). Quando a inseminação é realizada durante a fase em que o muco é de aspecto 
cristalino, Bonfert (1964), citado por Simplício (1987), recomenda uma segunda inseminação, 12 a 14 horas após a primeira. Nesse sentido, taxas de concepção de 76,2 e $86,4 \%$ foram observadas em cabras inseminadas quando o muco cervical apresentava-se com aspecto cristalino e estriado, respectivamente (França, 1981).

Diante desses resultados, enfatiza-se a necessidade de se observar o tipo de muco quando da inseminação artificial, e de se realizar nova inseminação, 8 a 12 horas após a primeira, na presença de muco com aspecto cristalino.

\section{CONCLUSÕES}

O estro pode ser eficientemente sincronizado por meio de duas aplicações de prostaglandina, intervaladas de 10 dias. O tipo de muco, observado durante a inseminação, influenciou a taxa de concepção, sendo o de aspecto estriado associado à melhor taxa de concepção; as taxas de concepção, associadas ao protocolo aqui aplicado, comprovaram a eficiência do container para o resfriamento do sêmen caprino; a categoria reprodutiva não exerceu influência sobre as taxas de concepção obtidas.

\section{AGRADECIMENTOS}

Os autores agradecem aos proprietários da Granja Água Limpa, localizada no município de Piau, Minas Gerais, pela cessão dos animais e das instalações, onde foi realizado este experimento.

\section{REFERÊNCIAS BIBLIOGRÁFICAS}

ALVES, N.G.; TORRES, C.A.A.; RODRIGUES, M.T. et al. Métodos de controle do estro e ovulação em caprinos. Cad. Tec. Vet. Zootec., n.48, p.61-70, 2005.

AMORIM, E.A.M.; TORRES, C.A.A.; FONSECA, J.F. et al. Sincronização de estro com CIDR reutilizado em cabras lactantes da raça Toggenburg tratadas com somatotropina bovina recombinante (r-bST). Arq. Bras. Med. Vet. Zootec., v.60, p.51-57, 2008.

ANUÁRIO ESTATÍSTICO DO BRASIL, Rio de Janeiro: IBGE, 2005. Disponível em: $<$ http://www.ibge.gov.br/>. Acessado em: 30 nov. 2006.
AZEVEDO, H.C.; MACHADO, R.; SIMPLÍCIO, A.A. Franqueamento cervical e taxa de concepção em cabras Moxotó inseminadas artificialmente com diferentes doses inseminantes. In: CONGRESSO BRASILEIRO DE REPRODUÇÃO ANIMAL, 11., 1995, Belo Horizonte. Anais...Belo Horizonte: CBRA, 1995. p.333.

AZEVEDO, O.I.; SIMPLÍCIO, A.A.; MACHADO, R. Influência do refluxo da dose inseminante sobre a fertilidade de cabras moxotó inseminadas artificialmente. In: REUNIÃO DA SOCIEDADE BRASILEIRA DE ZOOTECNIA, 35., 1998, Botucatu. Anais...Botucatu: SBZ, 1998. p.129-131.

BARBOSA, L.P. Avaliação de diferentes diluentes e métodos de congelamento de sêmen, em programas de inseminação artificial em caprinos da raça Alpina. 1999. 71f. Dissertação (Mestrado) - Universidade Federal de Viçosa, Viçosa.

BONFERT, A. Experiences dans l'insemination des chevres avec du sperm congelé. Elev. Insem., v.83, p.3-6, 1964.

CORTEEL, J.M. Production, storage and insemination of goat semen. In: SYMPOSIUM OF MANAGEMENT OF REPRODUCTION IN SHEEP AND GOATS, 1977, Madison. Proceedings...Madison: University of Wisconsin, 1977. p.41-57.

EVANS, G.; MAXWELL, W.M.C. Salamon's artificial insemination of sheep and goats. Austrália: Butterworths Pty Limited, 1987. 194p.

FONSECA, J.F. Controle e perfil hormonal do ciclo estral e performance reprodutiva de cabras Alpinas e Saanen. 2002. 122f. Tese (Doutorado) - Universidade Federal de Viçosa, Viçosa.

FRANÇA, M.P. Inseminação artificial com sêmen congelado de caprino no sertão do estado de Pernambuco. 1981. 59f. Dissertação (Mestrado) - Universidade Federal Fluminense, Niterói.

MAFFILLI, V.V.; TORRES, C.A.A.; BRUSHI, J.H. et al. Indução de estro em cabras da raça Toggenburg com dois diferentes dispositivos intravaginais. Arq. Bras. Med. Vet. Zootec., v.58, p.367-372, 2006.

MENCHACA, A.; RUBIANES, E. New treatments associated with timed artificial 
insemination in small ruminants. Reprod. Fertil. Develop., v.16, p.403-413, 2004.

OTT, R.S.; NELSON, D.R.; HIXON, J.E. Fertility of goats following syncronization of estrus with prostaglandin F2 $\alpha$. Theriogenology, v.13, p.341-345,1980.

PALHARES, M.S. Adequação de um novo container para o transporte do sêmen equino diluído e resfriado. I - Características termodinâmicas e funcionais, II - desempenho reprodutivo das éguas inseminadas. 1997. $246 \mathrm{f}$. Tese (Doutorado) - Escola de Veterinária, Universidade Federal de Minas Gerais, Belo Horizonte.

ROCA, J.; CARRIZOSA, J.A.; CAMPOS, I. Viability and fertility of unwashed MurcianoGranadiana goat spermatozoa diluted in Tris-egg yolk extender and stored at $5^{\circ} \mathrm{C}$. Small Rum. Res., v.25, p.147-153, 1997.
ROMANO, J.E. Effects of different stimuli of service on estrous duration in dairy goats. Theriogenology, v.42, p.875-879, 1994.

SAMPAIO, I.B.M. Estatística aplicada à experimentação animal. Belo Horizonte: FEPMVZ, 2002. 265p.

SIMPLÍCIO, A.A. Inseminação artificial na espécie caprina. Inf. Agropec., v.13, p.30, 1987.

SIQUEIRA, A.P. Inseminação artificial em caprinos com sêmen resfriado. 2006. $106 f$. Dissertação (Mestrado) - Escola de Veterinária, Universidade Federal de Minas Gerais, Belo Horizonte.

SIQUEIRA, A.P.; SILVA FILHO, J.M.; FONSECA, J.F. et al. Taxa de concepção de cabras inseminadas com sêmen caprino resfriado a $5^{\circ} \mathrm{C}$, por 12 ou 24 horas, em meio diluidor à base de gema de ovo. Arq. Bras. Med. Vet. Zootec., v.61, p.66-71, 2009. 